\title{
Natural Person-Following Behavior for Social Robots
}

\author{
Rachel Gockley \\ rachelg@cs.cmu.edu
}

\author{
Jodi Forlizzi \\ forlizzi@cs.cmu.edu \\ Carnegie Mellon University \\ 5000 Forbes Avenue \\ Pittsburgh, PA 15213
}

\author{
Reid Simmons \\ reids@cs.cmu.edu
}

\begin{abstract}
We are developing robots with socially appropriate spatial skills not only to travel around or near people, but also to accompany people side-by-side. As a step toward this goal, we are investigating the social perceptions of a robot's movement as it follows behind a person. This paper discusses our laser-based person-tracking method and two different approaches to person-following: direction-following and path-following. While both algorithms have similar characteristics in terms of tracking performance and following distances, participants in a pilot study rated the direction-following behavior as significantly more humanlike and natural than the path-following behavior. We argue that the path-following method may still be more appropriate in some situations, and we propose that the ideal personfollowing behavior may be a hybrid approach, with the robot automatically selecting which method to use.
\end{abstract}

\section{Categories and Subject Descriptors}

I.2.9 [Artificial Intelligence]: Robotics; J.4 [Social and Behavioral Sciences]: psychology

\section{General Terms}

Design, Human Factors, Experimentation, Performance

\section{Keywords}

Human-robot interaction, social robots, person tracking, person following

\section{INTRODUCTION}

Robots that operate around people in the real world need to move in coherent, easily-understood ways, so that they will not startle or harm the people around them. In particular, for robots that operate in hospitals or in nursing homes [5, 11], we argue that such robots need to move in ways that are socially acceptable to people - particularly to

Permission to make digital or hard copies of all or part of this work for personal or classroom use is granted without fee provided that copies are not made or distributed for profit or commercial advantage and that copies bear this notice and the full citation on the first page. To copy otherwise, to republish, to post on servers or to redistribute to lists, requires prior specific permission and/or a fee.

HRI'07, March 8-11, 2007, Arlington, Virginia, USA.

Copyright 2007 ACM 978-1-59593-617-2/07/0003 _..\$5.00.

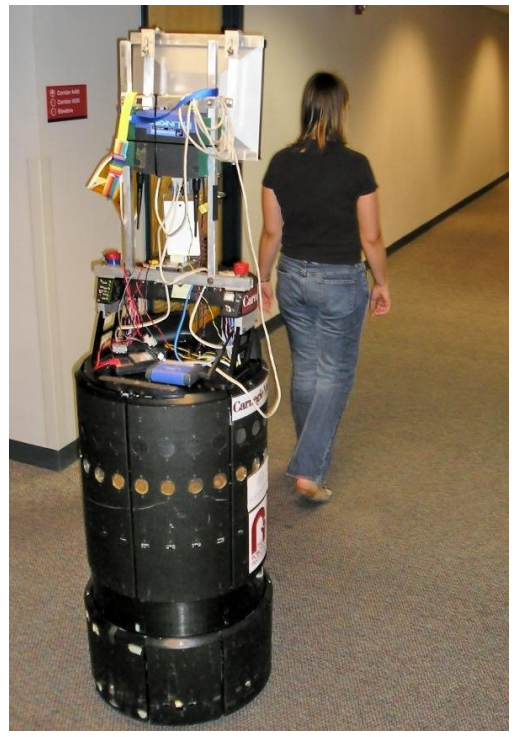

Figure 1: The robot, Grace, following a person down a hallway.

people who are not roboticists. Moving in easily understood and predictable ways will both improve people's trust in and comfort with the robot as well as help insure the safety of people moving near the robot.

Our overall goal is to develop robots with spatial social skills - robots that navigate in ways that people understand and expect. We are interested in developing robots that can operate as social assistants, such as encouraging nursing home residents to participate in social activities and accompanying them to common areas. Such a robot cannot simply drive toward a goal and expect the person to follow it; rather, it needs to engage the person and accompany him as a human would, traveling side-by-side with the person. Many social conventions exist for side-by-side accompaniment, and a robot must be able to obey these conventions. When two people walk next to each other, they may use cues such as utterances ("let's turn here"), gestures, and gross body movement into and away from each other's personal space. A robot also needs to offer and respond to these social cues, both verbal and non-verbal. In addition, when traveling next to a person, a robot must behave appropriately at bottlenecks, such as doorways or crowded hallways, when the robot may need to move ahead of, or follow be- 
hind, the person. Furthermore, the robot's behavior may need to be significantly different depending on the social situation, as side-by-side travel may take the form of purely social travel or of escorting with the robot either leading or following the person.

As a first step toward developing robots that can accompany people in socially acceptable ways, we are investigating social perceptions of a robot's movement as it follows behind a person (Figure 1), as a social assistant robot might when passing through doorways or navigating around obstacles. We have designed and tested two modes of person-following to determine which is more natural and socially acceptable. Participants in a pilot study agreed that the robot's behavior was more human-like when the robot always drove in the direction of the person, rather than when it followed the person's exact path. Thus, the "direction-following" method may be the more socially acceptable behavior for the robot to use. However, one can imagine situations when the path-following behavior may be more appropriate for the robot, such as in cluttered or unsafe environments where the robot's sensors may not be as reliable. We therefore propose a hybrid person-following model, and suggest that the robot may be able to learn the appropriate situations in which to use each method. Finally, we provide a discussion of what steps must be taken to move from a person-following robot to a robot that is capable of socially accompanying people side-by-side.

\section{RELATED WORK}

Person-tracking is currently an active area of research in robotics, and many person-trackers are demonstrated by having a robot follow the person being tracked. One of the more common methods of tracking people is to use a camera for face or color blob detection or for contour tracking [16]. Any such camera-based method, however, is prone to suffer from varying background colors and illumination conditions as a robot moves through various environments. Montemerlo and colleagues developed a laser-based persontracker for mobile robots [12]; however, their tracker requires the robot to have an a priori map of the environment, and will thus not work in a new or highly dynamic environment. Kluge et al demonstrated a method of object-tracking with a laser range-finder using network optimization techniques, but this method fails if any occlusions occur [9]. Several researchers have investigated combining camera-based and laser-based tracking methods, typically using a laser to find legs and a camera either to detect faces [8] or to track other visual regions of interest [10]. Using face detection, however, requires that the person always face the robot, and is thus awkward for the robot to follow behind or even walk next to a person. A final method of person-tracking requires attaching a tracking device to the person [2]. However, for such sensors to provide accurate location information, they are often large and therefore too awkward and intrusive to be worn. Our tracking approach is most similar to that of Topp et al [20], who use a single laser rangefinder and sample-based joint probabilistic data association filters to identify and track people [17].

In contrast with the above-mentioned literature, which focuses primarily on the technology of sensing and tracking a person, we are interested in people's perceptions of the robot's person-following behavior. Our research seeks to combine the technological aspects with social psychol-

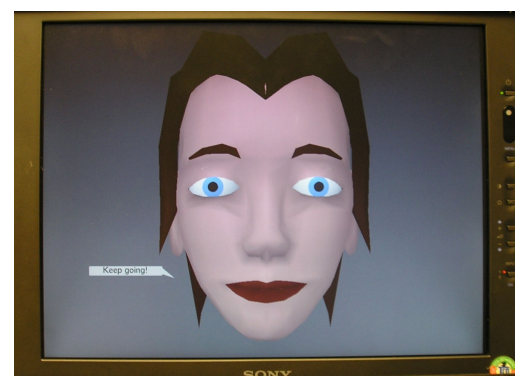

Figure 2: The LCD screen with graphical face used on the robot, shown here with a speech bubble that echos what the robot says ("Keep going!").

ogy and interaction design, to develop robots that can accompany people in socially acceptable ways. Even though some work has been done toward a robot that can navigate safely side-by-side with a person [15], such work also accounts only for the algorithms involved and does not consider human perception of the robot's behavior. In contrast, work that has focused on the social nature of navigation includes passing people in a hallway [14] and standing in line [13] in socially acceptable ways. Each of these studies has provided insight into people's perceptions of robotic behavior. We provide similar insight into people's perceptions of the specific behavior of person-following, and we hope to expand this knowledge into the general domain of spatial social human-robot interaction.

\section{DESIGN APPROACH}

We are interested in designing social behaviors for robots that allow people to feel comfortable in the robot's presence and to understand the robot's intentions.

The factors we considered in designing person-following behaviors for our robot include:

- Human-likeness: To what extent should the robot's behavior match that of a human in the same situation? The robot should behave according to people's expectations, but people may not expect a machinelike robot to act according to social conventions.

- Personal space: People determine how close they should be to one another according to societal conventions regarding personal space [7]. The robot must always remain at a distance that does not make the person uncomfortable.

- Reliability: The robot and its sensors must be capable of tracking a person with a high degree of reliability in order to remain useful and not frustrate the person.

- Safety: The robot must ensure the person's safety at all times; in particular, the robot must maintain enough space between itself and the person so as to avoid collisions.

\subsection{Hardware}

Our research platform for this work is Grace [19], an RWI B21 base with an LCD "head" mounted on top, as shown in Figure 1. With the head, the robot is roughly humanheight. The robot uses one primary sensor, a SICK LMS200 
scanning laser range-finder, mounted approximately $40 \mathrm{~cm}$ above the ground. The robot can move at speeds of up to $90 \mathrm{~cm} / \mathrm{s}$, but we tend to limit the speed to no more than $70 \mathrm{~cm} / \mathrm{s}$ due to safety concerns.

The robot's LCD screen is used to display an expressive, graphical face (Figure 2), which has been shown to encourage human interaction with the robot [3]. The robot is capable of speech via a synthesized voice and a text-to-speech system. The robot's face automatically lip-syncs with the speech.

\subsection{Person tracking}

We would like the robot to track people who may be moving or stationary in unknown, potentially dynamic, indoor environments. While our tracker is similar to that of Topp et al [20], we present the details of our particular implementation here. Briefly, each scan from the laser is segmented into person-sized blobs, which are tracked using individual particle filters [1] for each blob. The basic algorithm we use is as follows:

1. Since the robot - and hence the laser - may be moving, the particles being tracked are first transformed into the robot's current frame of reference. Updating the old information into the new frame is preferable to working in absolute coordinates, as odometry errors are not compounded over time.

2. The laser scan is next divided into segments. Adjacent points in the scan are considered part of the same segment if they are less than $10 \mathrm{~cm}$ apart.

3. Segments that contain any points further away than some threshold for tracking (we use $3.5 \mathrm{~m}$ ) are discarded.

4. Segments with a width (straight-line distance between the two endpoints) that is greater than $60 \mathrm{~cm}$ or less than $5 \mathrm{~cm}$ are discarded, as such measurements are unlikely to correspond to people.

5. Remaining segments that are greater than $20 \mathrm{~cm}$ are classified as a potential person. Smaller segments may be individual legs, and so we perform rudimentary clustering of these potential legs. If two such "leg" segments are separated by less than $40 \mathrm{~cm}$, they are classified as a single person. If no second leg is close enough to some segment, that segment is considered a potential person by itself.

6. All potential persons are tracked with a standard particle filter algorithm, using one filter for each person and 100 particles per filter. We use a Brownian (random) model of movement to predict where each segment might travel, as we found that any more sophisticated motion model could not account as well for a person's sudden stops or turns. Each filter is assigned to the closest potential person within $40 \mathrm{~cm}$ of the filter's center, and a new filter is created for any potential person that is more than $40 \mathrm{~cm}$ away from any unassigned filter.

7. Filters may be unassigned for up to 5 cycles of the tracker, after which they are removed. Allowing filters to remain unassigned helps to account for short occlusions, such as a person walking quickly past the person or object being tracked.

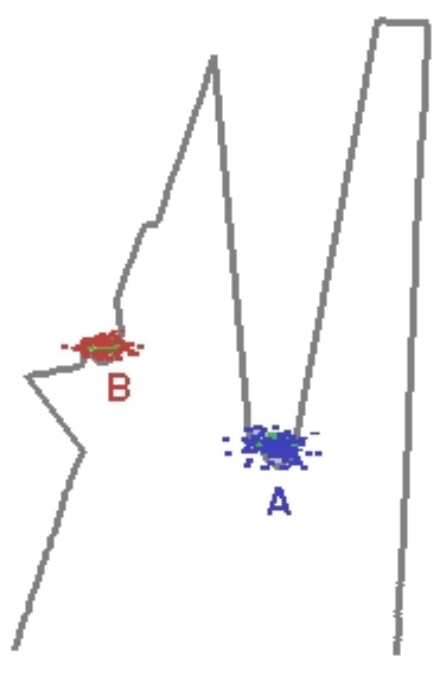

Figure 3: A sample scan from the laser range-finder, taken in a hallway, and overlaid with samples from the person tracker. The centermost samples (labeled A) correspond to the person being tracked and followed; the leftmost samples (labeled B) correspond to clutter in a doorway.

This tracking method, unlike most vision-based trackers, is relatively robust to the person's orientation; people can be tracked walking toward, away from, or past the robot. As such, this method can be used to track people in front of the robot, for following behind them (as discussed below), or to track people next to the robot, for side-by-side accompaniment or escorting.

An example laser scan with identified objects marked is shown in Figure 3. Note that this method of tracking identifies any "person-sized" objects as people, including objects such as chairs and garbage cans. However, without the use of additional sensors, such as vision, differentiating between a stationary person and similarly shaped inanimate objects is nearly impossible. Since we wish to track even people who are not moving, we chose to allow the tracker to identify other objects as people. Truly social interaction with people will require a more robust method that can distinguish people from inanimate objects; however, this tracker is sufficient for the purposes of following behind a person.

\subsection{Person following}

We designed two methods for following a person. The simplest method is to have the robot always attempt to drive directly toward the person's location. From general observations, we suspect that this is how people most often follow other people. This method often results in the follower cutting corners and generally not following in the exact footsteps of the leader. The second method, then, is to have the robot attempt to follow the exact path that the person took. While this method may not be the most human-like method, we hypothesized that it may better match people's expectations for a machine-like robot. For example, if a person is leading a robot somewhere, any step in the person's path may be taken for reasons that the robot does not know, 
Table 1: Vocalizations used by the robot during person-following.

\begin{tabular}{|c|c|c|}
\hline Vocalization type & When used & Examples \\
\hline Instructions & Initial detection of person & "Start walking, and I will follow you." \\
\hline Suggestions & Person has stopped moving & "Don't stop!", "Why are you stopping?" \\
\hline Encouragement & Randomly during travel & "Keep it up!", "You're doing great!" \\
\hline Error & Lost track of person & "I've lost you!" \\
\hline
\end{tabular}

and thus following the person's exact path may be the more appropriate behavior. Using the person-tracker described above, we have implemented both of these methods.

In both methods, the robot begins to follow a person as soon as someone is detected within $125 \mathrm{~cm}$ of the robot, in a cone of \pm 0.5 radians, as measured from the average location of a particle filter's samples. The robot then attempts to remain a constant distance $(120 \mathrm{~cm}$, plus or minus $10 \mathrm{~cm})$ from the tracked person. This is achieved through a simple proportional feedback control loop based on the error between the robot's current distance from the person and its desired position. Additionally, the change in range error over time is used to reduce oscillations in the system. Specifically, if the robot is too far from the person and the range error has increased (meaning the robot is falling further behind), then the velocity is increased based on the error; if the robot is too close and is getting closer, then the velocity is similarly decreased. The robot stops if the distance to the person drops below $90 \mathrm{~cm}$. The robot's maximum velocity is capped at $70 \mathrm{~cm} / \mathrm{s}$, due to safety concerns.

Currently, the distance at which the robot tries to follow is held constant, and is designed to keep the robot just outside of one's personal space. As several studies have found, the appropriate distance may vary according to an individual's personality traits $[21,6]$. We have not tested the personfollowing with different distances at this time.

The two person-following methods differ in how they select the robot's direction of travel. These differences, as well as social aspects of the robot's behavior, are discussed in the following sections.

\subsubsection{Direction-following}

In this method of following a person, the robot simply attempts to drive in the direction of the tracked person's current position. This is combined with the underlying obstacle avoidance control system by setting these goal directions using the Curvature-Velocity Method (CVM) [18]. With this method, the robot is able to follow the person through doorways and around corners without collisions.

It is interesting to note that person-following and most obstacle avoidance methods are fundamentally at odds, since following a person requires the robot to drive straight toward something that would normally be interpreted as an obstacle. To convince the Curvature-Velocity method to follow a person, we weighted the CVM parameters to strongly favor the goal direction over the preferred distance from obstacles and the preferred maximum speed. That is, the robot will favor going slowly close to obstacles (such as the person) as long as its heading is correct.

\subsubsection{Path-following}

In this more sophisticated approach, the robot attempts to follow the path that the person took as closely as possi- ble, such as switching to the opposite side of the hallway at a certain location and driving around corners with the same curvature as the person's travel. Path-following is achieved in much the same way as direction-following, except that the robot's goal direction is chosen according to the Pure Pursuit path-following algorithm [4]. At each tracker cycle, the person's location is stored, building a history of the person's path. The robot's goal point is selected as the point at which the person was at the desired distance from their current position (that is, $120 \mathrm{~cm}$ prior on the person's path). As with direction-following, the CVM method is used to integrate obstacle avoidance with the person-following behavior.

In addition, the robot's goal direction is constrained such that the robot will never intentionally turn to a point at which it can no longer track the person. This is necessary because the robot does not have a full 360-degree sensor coverage, but means that the robot may not always follow the person's exact path, particularly if the person walks in a tight circle around the robot.

\subsubsection{Interaction and error recovery}

To make the robot's behavior more social and humanlike, it periodically offers suggestions and encouragement, using synthesized computer speech. Additionally, the robot will verbally notify the person if he or she is walking too quickly for the robot to follow, as an able-bodied person can easily out-pace the robot. Importantly, this vocalization is also used to aid error recovery. If the laser tracker loses the target person, the robot immediately stops and provides verbal notification. When this occurs, the robot also reverts to its initial state of waiting to detect a person immediately in front of the laser. Target reacquisition is usually achieved by the person taking a few steps back toward the robot. The robot's full set of vocalizations is given in Table 1 .

\section{PERFORMANCE}

The two person-following algorithms differ most noticeably when turning corners; the direction-following approach results in the robot rounding corners much more so than the person did, whereas the robot explicitly attempts to follow the same curvature as the person when using the pathfollowing approach. This distinction can be seen in Figure 4.

\subsection{Functionality}

We present here the results from several trial runs with each person-following algorithm. Trials were performed both at the robot's maximum speed and at slower speeds.

\subsubsection{Procedure}

All trials took place in office building hallways, with varying amounts of clutter. While other people occasionally passed by the robot, no occlusions were permitted between the robot and the person it was following. Care was taken 


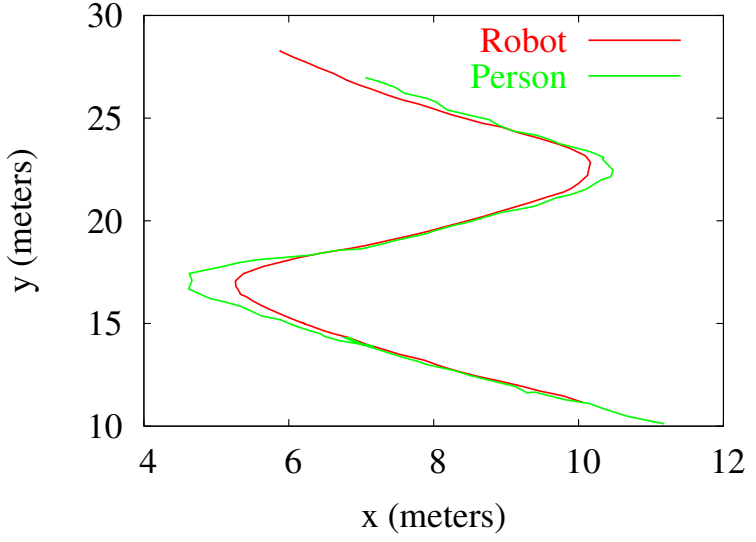

(a) Direction-following

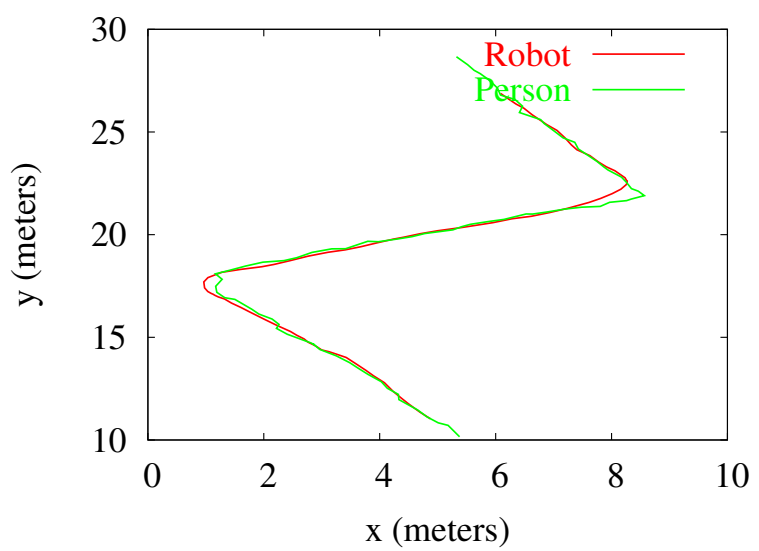

(b) Path-following

Figure 4: Paths of the person and the robot around corners, for each of the two approaches. The robot drastically cuts corners when not following the person's exact path. Note that each path shown is roughly $15 \mathrm{~m}$ in length.

to make the human's behavior as similar as possible across trial runs, though obviously no two runs with either algorithm were identical. The experimenter had prior knowledge of the robot's person-following behavior and did not attempt to "trick" the robot with sudden changes in movement patterns.

\subsubsection{Results}

Four trials with each approach were run at relatively high speeds. Each approach was run for a total of about 30 minutes (5-10 minutes per trial) and covered a total traversal of over 1 kilometer, with average speeds of close to the maximum allowed speed of the robot, $70 \mathrm{~cm} / \mathrm{s}$. On average, the robot was able to track the person over a distance of about 30 meters (1 minute) before an error in tracking occurred. Both approaches had a highest distance between tracking errors of over 160 meters (over 3.5 minutes). Using an analysis of variance (ANOVA), no significant difference was found between the two approaches in terms of the distance or time between tracking errors (distance $F[1,65]=0.79, p=0.3$; time $F[1,65]=0.27, p=0.6)$. In all cases of tracker failure, the robot was able to reacquire the person within moments of the person re-approaching the robot.

The tracking performs considerably better when the robot moves more slowly. When the person traveled at speeds of about $45 \mathrm{~cm} / \mathrm{s}$, the robot was able to follow for over 320 meters (over 10 minutes) before tracker failure, using each of the methods. During these slower traversals, the robot was able to remain on average approximately $1.3 \mathrm{~m}$ (standard deviation $25 \mathrm{~cm}$ ) from the person. Again, both personfollowing behaviors performed equivalently.

\subsection{User acceptance}

As discussed, one of our main interests is in people's perceptions of the different robot behaviors. We performed a pilot study to explore whether people prefer one personfollowing method over another.

\subsubsection{Procedure}

This study was performed during an informal gathering and had 10 participants ( 8 males, 2 females), including students, staff, and faculty members. Though all participants were experienced with robotics, few had advance knowledge of the nature of the study. Participants were asked to observe the robot's behavior as it followed the experimenter around the lab for several minutes. They then answered a short questionnaire on the robot's behavior, including whether the behavior met their expectations, how natural the behavior was, and how appropriate the robot's following and stopping distances were. This process was done once for each of the two person-following algorithms. To ensure that all participants viewed identical behaviors of the robot, all participants viewed both conditions as a single group; as such, conditions were not counter-balanced.

The robot followed the experimenter for about $50 \mathrm{~m}$ with each behavior. On average, the robot remained approximately $1.5 \mathrm{~m}$ from the experimenter during each trial. The experimenter stopped and started several times during each person-following method, so that participants could observe the robot's behavior both while moving and while stopped.

\subsubsection{Results}

Due to the within-subjects nature of this study, we analyzed the survey responses using paired $t$-tests across trials. Average responses and $t$-values for each question are given in Table 2.

Although the two behaviors are very similar, participants noticed their differences. Participants were asked to rate the robot's behavior according to whether it met their expectations ("not at all" (1) — "very much" (7)) and how natural the behavior was ("not at all" (1) - "human-like" (7)). As shown in Table 2, participants rated the robot's behavior as significantly more natural and human-like in the direction-following condition. In addition, participants felt that the direction-following robot behaved more according to their expectations. Notably, none of the participants rated the path-following behavior as better than the direction-following behavior on either of these questions. Furthermore, the answers to these two questions were highly correlated $(r=0.80, p<0.0001)$, indicating that participants expected the robot's behavior to be human-like, despite the robot's non-anthropomorphic physical shape.

Participants were also asked whether the robot followed and stopped at appropriate distances from the experimenter. 
Table 2: Average responses to the survey questions, with standard deviations given in parentheses. All questions were asked on scales of $\mathbf{1}-\mathbf{7}$. $N=10$.

\begin{tabular}{|l|c|c|c|}
\hline & \multicolumn{2}{|c|}{ Following Behavior } & Paired \\
Question & Direction & Path & $t$ \\
\hline Met expectations (not & 5.0 & 3.7 & $-4.33^{*}$ \\
at all-very much) & $(0.94)$ & $(0.95)$ & \\
\hline Natural (not at all- & 4.0 & 2.9 & $-3.97^{*}$ \\
human-like) & $(1.15)$ & $(0.88)$ & \\
\hline Following distance & 3.0 & 2.9 & -0.43 \\
(too close-too far) & $(0.94)$ & $(1.29)$ & \\
\hline Stopping distance & 4.9 & 5.4 & 1.86 \\
(too close-too far) & $(1.20)$ & $(1.71)$ & \\
\hline
\end{tabular}

* significant at $p<0.01$

They rated the distances on a scale of 1 ("too far away") to 7 ("too close"). Overall, participants felt that the robot stayed a little too far away from the experimenter while moving (overall mean 2.95, SD 1.10), but stopped at an appropriate distance (overall mean 5.15, SD 1.46). There were no significant differences in participants answers across the two person-following behaviors.

\subsection{Discussion}

Quantitatively, the two methods of person-following are equivalent; the behaviors do not differ in laser tracking performance, and both allow the robot to follow smoothly behind a person. The primary difference between the two behaviors occurs at corners, when the direction-following behavior causes the robot to curve much more gently than the person, given sufficient space to do so. If the following occurs in narrow corridors, the differences between the behaviors lessens, as obstacle avoidance constrains the robot's movement.

Qualitatively, however, people indicate that the directionfollowing behavior is significantly more human-like and more closely matches their expectations than when the robot follows the person's path. Several participants commented that, when performing path-following, the robot did not appear to react "quickly enough" to the person's turns (since the robot turned at the location where the person turned, rather than at the same time as the person), which may help explain this finding.

To date, we have performed only the small pilot study as described above. Obviously, many caveats apply to this sort of study, as the participants were all familiar with robots and most likely had very different expectations of robotic behavior than non-roboticists. However, while the participant population may have influenced the exact values of the survey questions, we expect that the relative differences between the different robot behaviors would be similar across other populations, as well. A further shortcoming of this study is that it analyzed only people's third-person observations of the robot's behaviors, and thus the results may not capture the full spectrum of people's preferences. However, while people's in situ experiences of a robot's behavior are clearly valuable, such testing is difficult to perform and evaluate when the robot's behavior occurs strictly behind - and thus out of sight of - the person.

\section{HYBRID APPROACH}

Our results indicate that the direction-following method is likely the most appropriate behavior in social settings, where a robot may be expected to behave in a human-like way. It would be easy to conclude from this that the directionfollowing method should always be used for social robots moving around people. However, many situations may call for the path-following approach. For example, while laser scanners are very useful and accurate sensors, they can not sense obstacles outside of their range, or obstacles that do not reflect light, such as glass doors and windows. In such situations, a robot might need to carefully follow a person's path in order to navigate such obstacles.

We propose that the best person-following behavior may be a hybrid approach, in which the robot can switch personfollowing modes as the situation requires. It may be possible for the robot to learn to recognize which behavior is appropriate - for example, if it begins to see a large amount of clutter or spurious sensor readings that might indicate unseen obstacles, the robot could switch to the more conservative path-following method.

This person-following behavior could be used in many situations. In particular, robotic assistants that accompany people between places may need to fall behind and follow a person through choke points, such as narrow corridors and doorways, and would need to do so in a socially acceptable way. A robot may be able to build a map more efficiently following a human leader than by randomly wandering in search of unmapped areas. In addition, robots that need to make regular trips between specific locations, such as medication deliveries in hospitals, could be taught the paths to take simply by following a human leader. We believe that the proposed hybrid approach may significantly increase the robot's robustness in situations such as these.

\section{FUTURE WORK}

In the work presented here, the robot has little to no understanding of the social cues that a person might use when leading a robot. That is, the robot responds only to the person's speed and location, ignoring aspects such as the person's identity or personality, spoken or gestured commands from the person, how and when to engage the person, appropriateness of behavior for the task (such as which person-following behavior to use when), or how to respond to other people in the vicinity.

This lack of understanding will only be amplified in a robot that accompanies a person side-by-side, as we are developing. Unfortunately, all of these aspects are currently open problems in the field of human-robot interaction. At a minimum, we intend to address the following in our future work:

- Better person-tracking. A sideways-facing laser rangefinder will allow the person-tracker described above to track people-shaped objects next to the robot, and we are currently investigating various laser configurations. To identify people, however, the tracker will likely need to be combined with a vision-based system for recognizing a person's face or clothing or by requiring the person to wear a visual fiducial or electronic tag.

- Generate appropriate social cues. We intend to study how people walk in pairs in order to gain a better 
understanding of what conventions people use when walking together, including interpersonal distance, use of gestures, and handling of obstacles and bottlenecks.

- Respond appropriately to unexpected behavior. If the person stops suddenly or turns unexpectedly, the robot should be able to react appropriately. This may require the robot to replan its path to follow the person or to interact with the person to determine the cause of his or her behavior.

Developing such social competencies will be a necessary step in allowing our person-following robot to travel sideby-side with a person in a socially acceptable manner.

\section{CONCLUSION}

We have presented a laser-based person-tracker and have described two different methods for a robot to follow a person: direction-following and path-following. We analyzed both behaviors and found that, while their quantitative performances are very similar, they are perceived differently by people. Specifically, observers of the robot's behaviors felt that the direction-following behavior appeared more natural and human-like, and more closely matched their expectations.

We believe that this study is a first step toward socially assistive robots that are capable of traveling with people in socially acceptable ways. We have shown that people's expectations for the robot were that it would move in a humanlike manner, following human social conventions. We intend to expand this research by studying further both how people walk in pairs and how people expect robots to navigate with people. Specifically, we plan to perform observational studies of how people walk together, develop both the hybrid person-following model described above and a model for side-by-side accompaniment, and perform extensive field testing with the robot. By developing robots that are capable of following human social conventions, we believe that we are making great strides toward robots that can function as social assistants for the elderly.

\section{ACKNOWLEDGMENTS}

This work was funded in part by an NSF Graduate Research Fellowship to the first author and by NSF grants \#IIS-0329014 and \#IIS-0121426.

\section{REFERENCES}

[1] S. Arulampalam, S. Maskell, N. Gordon, and T. Clapp. A tutorial on particle filters for on-line non-linear/non-gaussian bayesian tracking. IEEE Transactions of Signal Processing, 50(2):174-188, Feb 2002.

[2] R. Bianco, M. Caretti, and S. Nolfi. Developing a robot able to follow a human target in a domestic environment. In A. Cesta, editor, Proceedings of the First RoboCare Workshop, pages 11-14, Rome, Italy, Oct. 2003.

[3] A. Bruce, I. Nourbakhsh, and R. Simmons. The role of expressiveness and attention in human-robot interaction. In Proceedings of the IEEE International Conference on Robotics and Automation (ICRA), pages 4138-4142, May 2002.
[4] R. C. Coulter. Implementation of the pure pursuit path tracking algorithm. Technical Report CMU-RI-TR-92-01, Robotics Institute, Carnegie Mellon University, Pittsburgh, PA, Jan. 1992.

[5] J. F. Engelberger. Health-care robotics goes commercial: The Helpmate experience. Robotica, 11(6):517-523, Nov-Dec 1993.

[6] R. Gockley and M. Matarić. Encouraging physical therapy compliance with a hands-off mobile robot. In Proceedings of Human-Robot Interaction, pages 150-155, Mar 2006.

[7] E. T. Hall. The Hidden Dimension. Doubleday, New York, 1966.

[8] M. Kleinehagenbrock, S. Lang, J. Fritsch, F. Lömker, G. A. Fink, and G. Sagerer. Person tracking with a mobile robot based on multi-modal anchoring. In Proceedings of the 2002 IEEE Int. Workshop on Robot and Human Interactive Communication, pages 423-429, Berlin, Germany, Sept 2002.

[9] B. Kluge, C. Köhler, and E. Prassler. Fast and robust tracking of multiple moving objects with a laser range finder. In Proceedings of the IEEE International Conference on Robotics and Automation, pages 1683-1688, Seoul, Korea, May 2001.

[10] M. Kobilarov, G. Sukhatme, J. Hyams, and P. Batavia. People tracking and following with mobile robot using an omnidirectional camera and a laser. In Proceedings of the IEEE International Conference on Robotics and Automation, pages 557-562, Orlando, Florida, May 2006.

[11] M. Montemerlo, J. Pineau, N. Roy, S. Thrun, and V. Verma. Experiences with a mobile robotic guide for the elderly. In Proceedings of the National Conference of Artificial Intelligence (AAAI 02), pages 587-592, Edmonton,AB, July 2002.

[12] M. Montemerlo, S. Thrun, and W. Whittaker. Conditional particle filters for simultaneous mobile robot localization and people-tracking. In Proceedings of the IEEE Int. Conference on Robotics and Automation (ICRA), pages 695-701, Washington, DC, May 2002.

[13] Y. Nakauchi and R. Simmons. A social robot that stands in line. In Proceedings of the Conference on Intelligent Robots and Systems (IROS), pages 357-364, Oct 2000.

[14] E. Pacchierotti, H. I. Christensen, and P. Jensfelt. Human-robot embodied interaction in hallway settings: a pilot user study. In Proceedings of the IEEE International Workshop on Robots and Human Interactive Communication (RO-MAN), pages 164-171, Nashville, TN, 2005.

[15] E. Prassler, D. Bank, and B. Kluge. Key technologies in robot assistants: Motion coordination between a human and a mobile robot. Transactions on Control, Automation and Systems Engineering, 4(1):56-61, Mar. 2002.

[16] C. Schlegel, J. Illmann, K. Jaberg, M. Schuster, and R. Wörz. Vision based person tracking with a mobile robot. In Proceedings of the Ninth British Machine Vision Conference (BMVC), pages 418-427, Southampton, UK, 1998.

[17] D. Schulz, W. Burgard, D. Fox, and A. B. Cremers. 
Tracking multiple moving targets with a mobile robot using particle filters and statistical data association. In Proceedings of the IEEE Int. Conference on Robotics and Automation (ICRA), Seoul, Korea, 2001.

[18] R. Simmons. The curvature-velocity method of local obstacle avoidance. In Proceedings of the Intl. Conference on Robotics and Automation, Minneapolis MN, Apr. 1996.

[19] R. Simmons, D. Goldberg, A. Goode, M. Montemerlo, N. Roy, B. Sellner, C. Urmson, A. Schultz,

M. Abramson, W. Adams, A. Atrash, M. Bugajska, M. Coblenz, M. MacMahon, D. Perzanowski, I. Horswill, R. Zubek, D. Kortenkamp, B. Wolfe, T. Milam, and B. Maxwell. GRACE: An autonomous robot for the AAAI robot challenge. AAAI Magazine, 24(2):51-72, Summer 2003.
[20] E. A. Topp and H. I. Christensen. Tracking for following and passing persons. In Proceedings of the IEEE/RSJ International Conference on Intelligent Robots and Systems (IROS), pages 70-76, Edmonton, Alberta, Canada, Mar. 2005.

[21] M. L. Walters, K. Dautenhahn, R. te Boekhorst, K. L. Koay, C. Kaouri, S. Woods, C. Nehaniv, D. Lee, and I. Werry. The influence of subjects' personality traits on personal spatial zones in a human-robot interaction experiment. In Proceedings of CogSci-2005 Workshop: Toward Social Mechanisms of Android Science, pages 29-37, Stresa, Italy, Jul 2005. 